\title{
Carbon Sputtering Technology for MPGD detectors
}

\author{
Atsuhiko Ochi, Yasuhiro Homma, Yuji Yamazaki, Fumiya Yamane, \\ Tsuyoshi Takemoto \\ Kobe University \\ E-mail: ochi@kobe-u.ac.jp yasuhirohomma3@gmail.com. \\ yamazakiephys.sci.kobe-u.ac.jp fumiyamanedstu.kobe-u.ac.jp \\ tsuyoshiestu.kobe-u.ac.jp
}

Tatsuo Kawamoto, Yousuke Kataoka, Tatsuya Masubuchi, Yuki Kawanishi, Shingo Terao

University of Tokyo

E-mail: Tatsuo.Kawamotodcern.ch Yousuke.Kataokadcern.ch

tatsuya.masubuchiecern.ch yuki.kawanishiecern.ch,

shingo.teraodcern.ch

Carbon sputtering is a promising technology for making resistive electrodes for MPGDs (Micro Pattern Gaseous Detectors). The research on this technology started in the context of the development of MicroMEGAS detectors for the ATLAS muon system upgrade. By sputtering carbon layers of varying thickness (a few hundred to a few thousand angstrom) and doping the nitrogen, the surface resistivity can be controlled between $50 \mathrm{k} \Omega / \mathrm{sq}$. and $4 \mathrm{G} \Omega / \mathrm{sq}$. Several small $(10 \times 10$ $\left.\mathrm{cm}^{2}\right)$ and medium size foils $\left(50 \times 100 \mathrm{~cm}^{2}\right)$ have been produced and successfully tested. The carbon layer is deposited on thin ( 25 to $50 \mu \mathrm{m}$ ) insulating foils (e.g., Kapton) by sputtering resulting in a good uniformity $(<30 \%)$ of the resistivity. Extremely fine electrodes structures $(<50 \mu \mathrm{m})$ can be achieved using the liftoff process technique. The foils produced so far are extremely robust with respect to mechanical and chemical damage. We report on the fabrication technique and the performance in operating detectors. Given the positive experience with MicroMEGAS detectors we also address other possible applications that could be interesting for MPGD detectors, e.g., Micro Pixel Chambers, GEMs, etc.

Technology and Instrumentation in Particle Physics 2014,

2-6 June, 2014

Amsterdam, the Netherlands

\footnotetext{
${ }^{*}$ Corresponding Author.
} 


\section{Introduction}

The MPGD (MicroMEGAS, GEM, $\mu$-PIC, etc.) are very promising particle detectors which provides both fine granularity and wide detection area. The electric discharge problem has been very critical problem in MPGDs, however it was almost overcome for using high resistive electrodes [1] 3]. As for the resistive material, generally the surface resistivity of around $100 \mathrm{k} \Omega / \mathrm{sq}$. $-1 \mathrm{G} \Omega / \mathrm{sq}$. are required. We are proposing a novel method for making a resistive pattern for MPGDs, using the carbon sputtering with lift off process, instead of the standard carbon black loaded material. The large foil, more than a few $\mathrm{m}^{2}$, can be made in industrial sputtering process. It meets the requirements for HEP experiments, which need large size detectors. This paper discusses briefly the carbon sputtering foil development for mainly ATLAS MicroMEGAS for LHC upgrade [4]. The prototype production of small test chamber and large $(\sim 1 \mathrm{~m})$ MicroMEGAS foils, and resistivity control are described. Details of those results and other studies for resistive foils will be presented in a forthcoming paper.

\section{Sputtering and liftoff technique}

The sputtering technology is now commonly used for producing thin metal or non-metal foils. We have used a large industrial sputtering chamber in Be-Sputter Co., Ltd. Using a rotating drum, the $4.5 \mathrm{~m} \times 1 \mathrm{~m}$ foil can be used as a substrate. The carbon is sputtered on the substrate as an amorphous diamond like carbon (a-DLC) using graphite sputtering target. To make the structure of the MPGD electrodes, the liftoff process is combined. Firstly, the negative pattern of the electrodes image is formed by photo resist, as the usual PCB (printed circuit board) production technique. Next, the carbon is sputtered on the surface, and then, the resists are removed chemically. The liftoff is processed by the PCB industrial company, Raytech Inc. The precisions of the sputtering pattern is determined by photo resist imaging. A few tens micrometer accuracy is possible for that precision using the commonly used industrial PCB process.

Prototypes of MicroMEGAS with resistive strips were produced and tested. The small prototype with $10 \mathrm{~cm} \times 10 \mathrm{~cm}$ detection area has been made, as shown in figure 1 Gas gain above 10000 was observed using $\operatorname{Ar}(85 \%)+\mathrm{CO}_{2}(15 \%)$ mixed gas. Operations in fast neutron condition $(\sim 2$

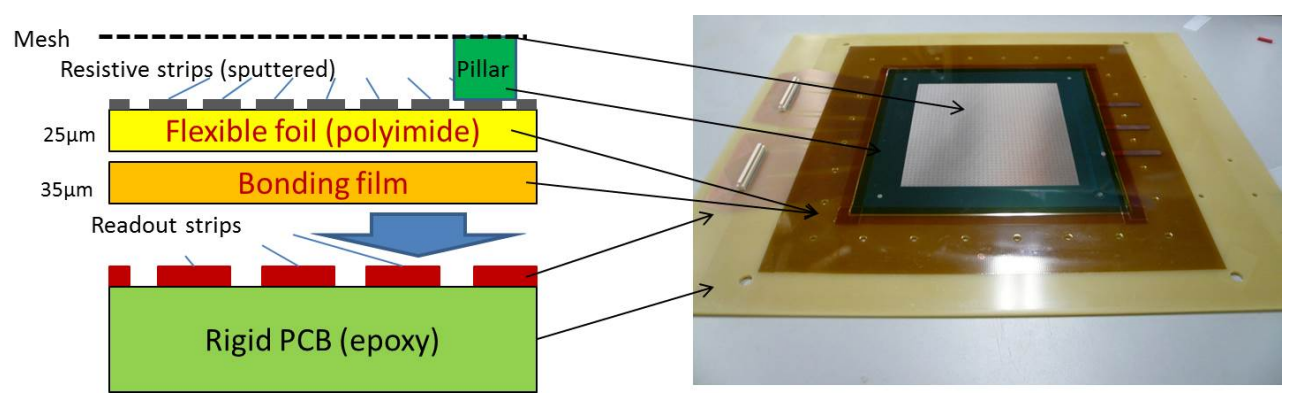

Figure 1: Schematic cross section of the $10 \mathrm{~cm} \times 10 \mathrm{~cm}$ resistive MicroMEGAS with sputtered carbon, and the picture of a prototype chamber. 

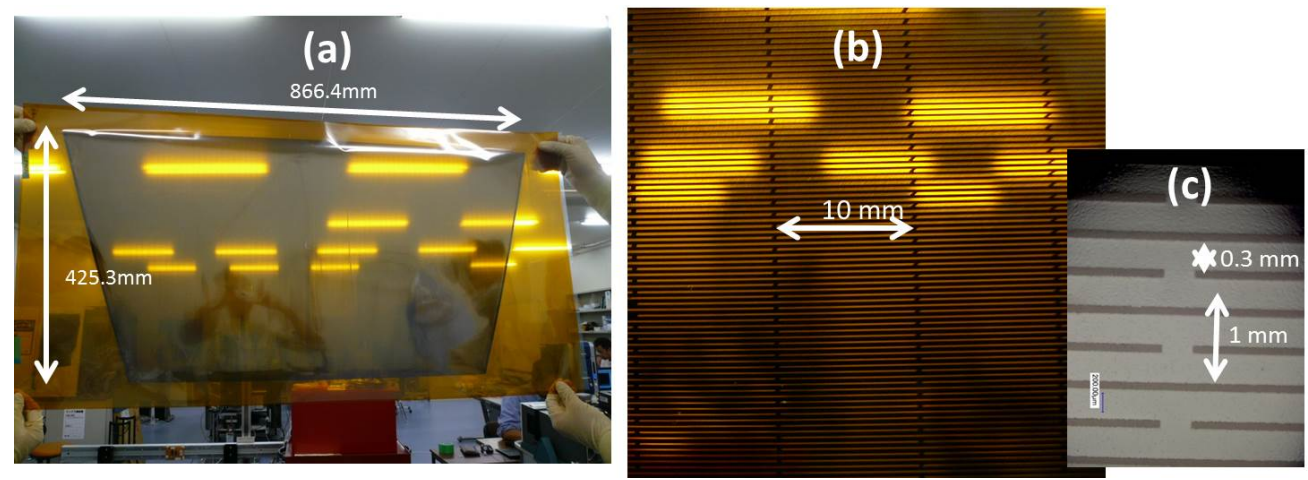

Figure 2: Schematic cross section of the $10 \mathrm{~cm} \times 10 \mathrm{~cm}$ resistive MicroMEGAS with sputtered carbon, and the picture of a prototype chamber.

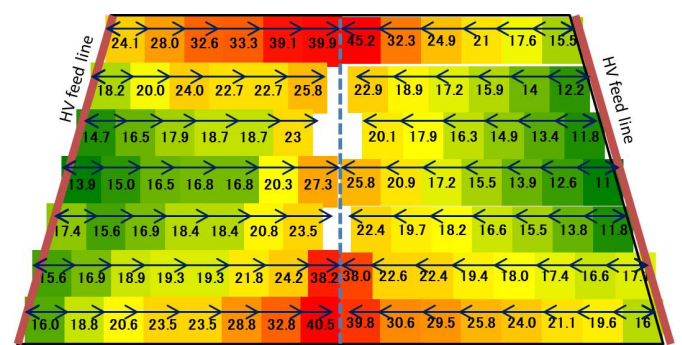

Figure 3: Resistivity map for large size resistive strip foil. The resistivity from HV feed line (both inclined side) were measured for each $5 \mathrm{~cm}$ point. Values are in $\mathrm{M} \Omega$. The predicted resistive value is not calculated yet, but this result agree well with approximate estimates, and the uniformity is roughly estimated to be below $30 \%$ [6.

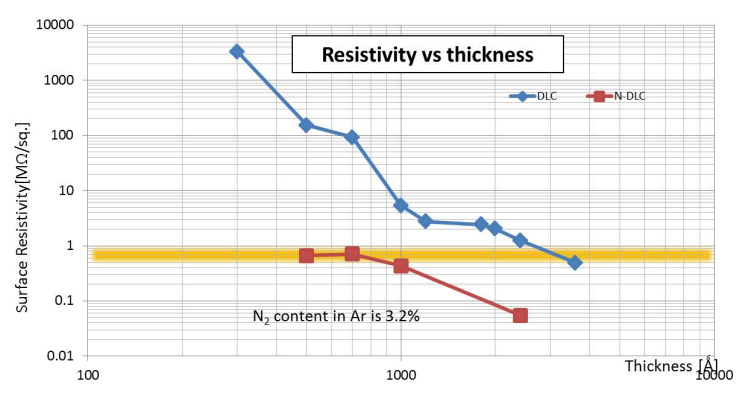

Figure 4: Surface resistivity as a function of the sputtered carbon thickness. The blue line shows the resistivity of pure carbon sputtering, and the red line shows that of Nitrogen doped foils. The orange band represents the required resistivity for the ATLAS MicroMEGAS.

$\mathrm{MeV}$ neutron, $10^{6} \mathrm{n} / \mathrm{sec} / \mathrm{cm}^{2}$ ) were also tested, and stable operation and spark reduction were confirmed. No damage was observed after the detector operation of more than $10^{10}$ neutron $/ \mathrm{cm}^{2}$ [5]. Large size foils for the ATLAS MicroMEGAS prototype were also produced. Figure 2 shows a pictures of the large foil (a), a magnified picture (b) and a microscopic picture (c). The trapezoidal shape of $85 \mathrm{~cm} \times 45 \mathrm{~cm}$ with $415 \mu \mathrm{m}$ pitch strips were produced successfully. The uniformity of the resistivity was measured for this large foil. Figure 3 shows the measured resistivity from the inclined side, which is designed as high voltage provider. The results agree well with the calculated values within $30 \%$. Total 8 foils were produced for ATLAS MicroMEGAS prototype chamber [7], and being tested.

\section{Resistivity control}

The resistivity of the carbon sputtered foil is dependent on the carbon thickness. The surface resistivity for carbon thickness of $30 \mathrm{~nm}$ is about $4 \mathrm{G} \Omega / \mathrm{sq}$., and the thickness of $360 \mathrm{~nm}$ is 
$500 \mathrm{k} \Omega / \mathrm{sq}$. Figure 4 shows the surface resistivity dependance on the carbon thickness from our measurement. ATLAS MicroMEGAS require the surface resistivity of around $700 \mathrm{k} \Omega / \mathrm{sq}$., and carbon thickness of $300 \mathrm{~nm}-360 \mathrm{~nm}$ was required. In other hands, the deposition speed of carbon is very slow. In the case of our production, the maximum deposition speed using single target is $30 \mathrm{~nm} /$ hour. The deposition time for producing the prototype foils in figure 2 a) was 6 hours, which is too long for mass production.

We have developed a new method for reducing the surface resistivity with shorter deposition time. To reduce the surface resistivity with shorter deposition time, we have conceived to dope the nitrogen into the carbon foil. This idea originates from the analogy with n-doped semiconductors. Generally, the molecular structure of the sputtered carbon is a-DLC, which is characterized by a low amount of charge carriers. Nitrogen doping is to increase carrier electrons. We have mixed the nitrogen gas into the Argon gas while sputtering process. In our experience, where carbon was sputtered with nitrogen gas of $3.2 \%$, we got resistive foil of $400 \mathrm{k} \Omega / \mathrm{sq}$. with $100 \mathrm{~nm}$ thickness. This resistivity is ten times smaller than pure carbon sputtering case. The measured resistivity with $3.2 \%$ nitrogen gas mixing is shown in figure 4 From those results, we can get the appropriate surface resistivity for ATLAS MicroMEGAS with only $70 \mathrm{~nm}$ thickness, with which the deposition time is about 40 minutes using double station of carbon targets.

\section{Summary}

The carbon sputter and liftoff method is newly developed for producing MPGD resistive electrodes. Our tests show very promising results, and many other measurements e.g. mechanical tolerance tests, chemical tests, high voltage breakdown tests and detailed results of above tests will be detailed in a forthcoming publication.

\section{Acknowledgments}

We would like to thank H. Uehara of Raytech-Inc. and T. Date of Be-Sputter Co. Ltd., whose diligent efforts contributed to our proposal and improved the fabrication process. We thanks to ATLAS NSW MicroMEGAS group members, especially J. Watchack and F. Kuger for many discussions and measurements. We also thank the RD51 members, especially Rui de Oliveira, for many fruitful suggestions. This research was supported by JSPS/MEXT Grants-in-Aid for Scientific Research (KAKENHI No.23340072, 24654067, 26104707).

\section{References}

[1] R. Oliveira et al., IEEE Trans. on Nucl. Sci. Vol. 57, No. 6 (2010)

[2] R. Oliveira et al., arXiv:1101. 3727, (2011)

[3] A. Ochi et al., JINST 9 C01039, (2014)

[4] T. Kawamoto et al., New Small Wheel Technical Design Report, CERN-LHCC-2013-006, (2013)

[5] Details will be shown in forthcoming paper in preparation.

[6] The results of measurements are shown by F. Kuger, in the ATLAS MicroMEGAS meeting.

[7] P. Iengo, in this volume of proceedings. 